\title{
Microwave synthesis of hematene and other 2D oxides
}

\section{Sumit Chahal ${ }^{1}$, Susan M. Kauzlarich ${ }^{2, *}$ and Prashant Kumar ${ }^{1,3,{ }^{*}}$}

1Department of Physics, Indian Institute of Technology Patna, Bihta Campus, Patna-801106, ${ }^{2}$ Department of Chemistry, University of California, Davis, CA-95616, USA.

${ }^{3}$ Birck Nanotechnology Centre, Purdue University, West Lafayette, IN-47907, USA.

a

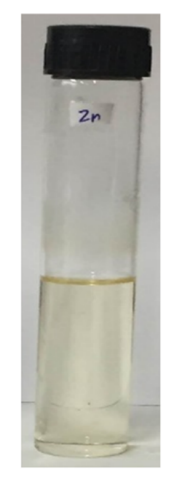

d

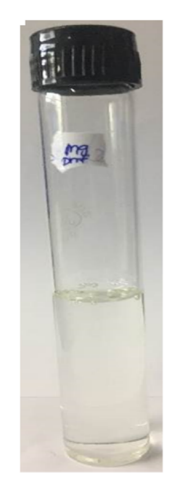

b

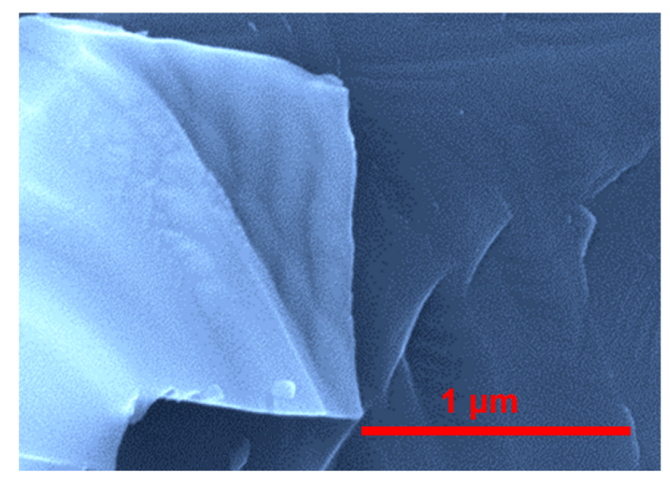

e

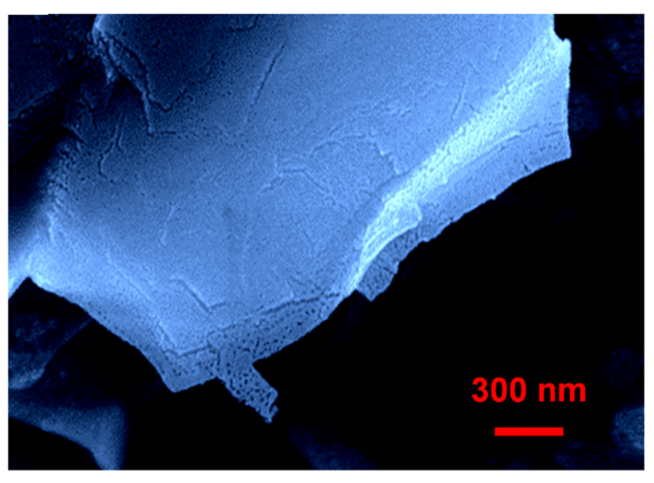

C

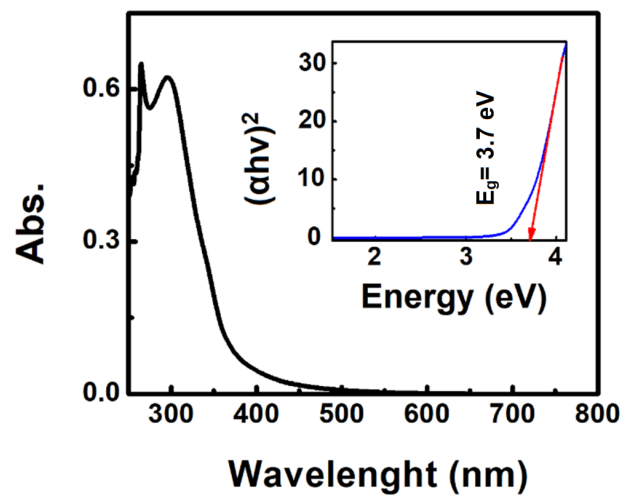

f

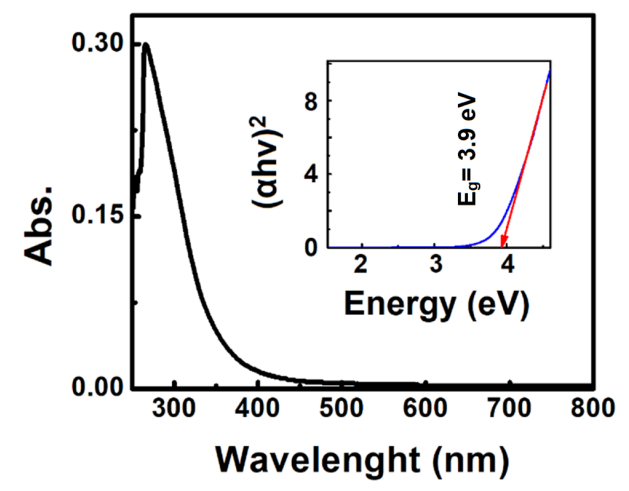

Fig S1. (a) Digital image, (b) FESEM and (c) Tau plot of 2D zinc oxide having band gap of $3.7 \mathrm{eV}$. (d) Digital image, (e) FESEM and (f) Tau plot of 2D magnesium oxide having band gap of $3.9 \mathrm{eV}$. 